\title{
A implementação do sistema de contabilidade de custos nos municípios portugueses
}

The implementation of the cost accounting system in the portuguese municipalities

La implementación del sistema de contabilidad de costes en los municipios portugueses

\section{Ana Luísa Pereira}

Mestre em Administração Pública pela Universidade de Lisboa, Instituto Superior de Ciências Sociais e Políticas

Endereço: Rua Almerindo Lessa - 1300-663 - Lisboa - Portugal

E-mail: anapereira 2@hotmail.com

Telefone: $(+351) 213619430$ - Ext. 453168

\section{Ana Lúcia Romão}

Doutora em Economia pela Universidade do Minho

Professora Auxiliar no Instituto Superior de Ciências Sociais e Políticas da Universidade de Lisboa

Endereço: Rua Almerindo Lessa - 1300-663 - Lisboa - Portugal

E-mail: anaromao@iscsp.ulisboa.pt

Telefone: (+351) 213619430 - Ext. 453168 | (+351) 967608180

Artigo recebido em 12/06/2015. Revisado por pares em 10/12/2015. Reformulado em 31/12/2015. Recomendado para publicação em 30/01/2016 por Sandra Rolim Ensslin (Editora Científica). Publicado em 30/03/2016. 


\title{
Resumo
}

As fortes restrições orçamentais têm vindo a pressionar os municípios a aumentar os índices de eficiência, de eficácia e de qualidade no serviço prestado e os sistemas de contabilidade de custos (SCC) devem desempenhar um papel preponderante nesta matéria. Neste contexto, o presente estudo tem como objetivos principais avaliar a implementação do SCC nos municípios portugueses, bem como, analisar a utilidade da informação produzida por este sistema. Para tal, elaborámos um questionário (distribuído pelos 308 municípios), o qual foi utilizado como ferramenta na operacionalização da investigação. Os resultados demonstraram que apenas 58\% dos inquiridos já implementou o SCC encontrando-se numa fase de implementação intermédia. Quanto aos outputs produzidos pelo sistema, concluímos que, apesar dos mesmos assumirem um papel importante para os inquiridos, têm ainda uma utilização bastante reduzida, não contribuindo de forma efetiva para a gestão municipal e para o controlo e racionalização dos recursos dos municípios.

Palavras-chave: Contabilidade pública. Sistema de contabilidade de custos. Plano oficial de contabilidade das autarquias locais. Municípios.

\begin{abstract}
The severe budget constraints have been pressing municipalities to increase the levels of efficiency, effectiveness and quality of service and the cost accounting systems (CAS) should develop a leading role in this matter. In this context, the present study's main objectives are to analyze the implementation of the CAS in the Portuguese municipalities, and to evaluate the usefulness of the information produced by this system. Thus, we developed a questionnaire (distributed to 308 municipalities), which was used as a methodological tool for conducting research. The results showed that only $58 \%$ of respondents have already implemented the CAS, lying in an intermediate implementation phase. As regards the outputs produced by the system, we conclude that, despite the respondents considered important to adopt the CAS as a transparency tool and to support decision making, they still have a limited use, not contributing effectively to municipal management, control and rationalization of municipalities' resources.
\end{abstract}

Keywords: Public accounting. Cost accounting system. Official accounting plan for local government. Municipalities.

\section{Resumen}

Las restricciones presupuestarias han estado presionando a los municipios para elevar los niveles de eficiencia, eficacia y calidad del servicio prestado y los sistemas de contabilidad de costes (SCC) debem desempeñar un papel destacado en este sentido. El objetivo principal de este estudio consiste en evaluar la aplicación del SCC en los municipios portugueses, así como evaluar la utilidad de la información producida por este sistema. Con este fin, hemos preparado un cuestionario (distribuido a 308 municipios), que ha sido utilizado como herramienta para la operacionalización de la investigación. Los resultados han demostrado que sólo el 58\% de los inquiridos han implementado el SCC, encontrándose en una fase de ejecución intermedia. Relativamente a los resultados generados por el sistema, llegamos a la conclusión de que tienen un uso muy limitado y no contribuyen eficazmente a la gestión municipal ni al control y la racionalización de los recursos de los municipios.

Palabras clave: Contabilidad pública. Sistema de contabilidad de costes. Plan oficial de contabilidad para la administración local. Municipios. 


\section{Introdução}

A conjuntura económica dos últimos anos, o cenário de escassez de recursos e a necessidade cada vez mais premente de ter um conhecimento claro e rigoroso dos custos das atividades e dos serviços, têm vindo a impulsionar a necessidade de recorrer a instrumentos que apoiem a gestão na tomada de decisão, que racionalizem os recursos públicos, que propiciem o controlo rigoroso dos custos e aumentem a responsabilidade inerente ao dever da prestação de contas (accountability) e da análise custo-benefício (value for money), como é o caso do Sistema de Contabilidade de Custos (SCC). A contabilidade de custos tem como objetivo principal proporcionar informação relevante para a tomada de decisão (KAPLAN; NORTON, 1992).

A implementação do SCC no Setor Público Administrativo (SPA) enquadra-se no modelo de adoção de técnicas de gestão privada que ocorreu no âmbito da reforma administrativa subjacente ao paradigma da Public Management. Em Portugal, no caso dos municípios, a implementação do SCC foi impulsionada pela publicação de vários diplomas e a sua obrigatoriedade foi instituída pela entrada em vigor do Plano Oficial de Contabilidade das Autarquias Locais (POCAL), em 2002.

Esta problemática levou-nos a desenvolver uma pesquisa empírica que permitisse evidenciar a fase de implementação do SCC nos municípios portugueses e o seu contributo para a gestão municipal.

O estudo pretende verificar se o SCC já se encontra implementado nos municípios portugueses, determinando a sua fase de implementação, e se os outputs produzidos por este sistema estão a ser utilizados no sentido de fomentar a qualidade, a transparência e a accountability na gestão municipal e a garantir a íntegra gestão dos recursos públicos numa lógica de economia, eficiência e eficácia.

De modo a operacionalizarmos a investigação, elaborámos um questionário que foi distribuído pelos 308 municípios que compõem a população em análise. A aplicação do questionário teve como objetivos, caracterizar os municípios respondentes, identificar as determinantes e os constrangimentos da implementação do SCC, aferir a fase em que se encontra a implementação do SCC, averiguar as regras que estão a ser seguidas na implementação do SCC, e se as mesmas estão de acordo com as normas definidas no POCAL, e determinar a utilidade retirada do SCC em termos da aplicação dos outputs produzidos pelo sistema.

O principal contributo deste estudo resulta do trabalho empírico desenvolvido e das conclusões que a partir do mesmo se obtiveram, nomeadamente a identificação das principais dificuldades apontadas para a não implementação do sistema ou para a não utilização da informação financeira que o sistema disponibiliza.

\section{A Contabilidade de Custos nos Municípios}

A introdução da contabilidade no SPA, nomeadamente nos municípios, tem como finalidade assegurar a concretização do "princípio da responsabilidade orçamental", ou seja, assegurar que os órgãos competentes para o efeito (Governo e Administração Pública) respondam perante os cidadãos no momento da elaboração do orçamento (CAIADO; PINTO, 2002) e ocorreu num contexto de desenvolvimento de novas técnicas de gestão no setor

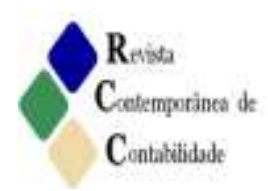


público e de reforma administrativa, impulsionado pelo fenómeno da globalização e pelo despoletar da era das tecnologias de informação. As organizações públicas têm de ser responsáveis quer ao nível dos recursos públicos (comuns a todas as pessoas) quer ao nível dos resultados que pretendem alcançar (REGINATO, 2010). Neste sentido, Burns e Scapens (2000) sublinham que o sistema de contabilidade deve permitir a satisfação de novas necessidades de informação por parte dos utilizadores, contribuindo desta forma para a melhoria do desempenho organizacional.

Ao longo das últimas décadas, o setor público tem sido objeto de uma reavaliação profunda em muitos países, com a introdução de novos programas, políticas e reformas (BARRETTA; BUSCO, 2011, JACKSON; LAPSLEY, 2003), enquadrados no modelo da Nova Gestão Pública (NGP) que assumiu uma importância crescente desde o final dos anos 70 nos países da OCDE, constituindo o centro da agenda burocrática em alguns destes países, e que se caracterizou pela importação de conceitos e técnicas do setor privado para o setor público (HOOD, 1991, 1995; POLLITT, 2006). No entender de Nyland e Pettersen (2004) a NGP é um modelo de decisão racional, alicerçado nos conceitos de eficiência, eficácia e economia, onde os sistemas de gestão, auditoria e transparência introduzem ganhos de informação significativos, incutindo no setor público uma maior responsabilidade e transparência (accountability). A NGP pretendeu esbater as diferenças entre o setor privado e o setor público, incidindo numa maior e melhor prestação de contas (HOOD, 1995), o que, consequentemente, levou à substituição de abordagens orientadas por processos por abordagens que enfatizam os resultados (LIGUORI, 2012). Van Helden, et al. (2010) referem que no setor público foram seguidas novas técnicas de gestão e de contabilidade, precisamente como uma resposta à crítica de que o setor público, nas últimas décadas, tem sido insuficientemente eficaz e eficiente. Por outro lado, Lapsley (2009) questiona a eficácia deste modelo devido às diferenças existentes entre o setor público e o setor privado e Pettersen (2001) destaca que o debate internacional tem vindo a centrar-se sobre os efeitos da NGP e a falta de ganhos de eficácia destas reformas.

Em Portugal, a introdução de um novo sistema de contabilidade no setor público foi materializada através da Lei de Bases da Contabilidade Pública (Lei n. ${ }^{\circ}$ 8/1990, de 20 de fevereiro), que estabeleceu o regime financeiro dos organismos e serviços da Administração Central e do Plano Oficial de Contabilidade Pública (Decreto-Lei n. ${ }^{\circ}$ 232/1997, de 3 de setembro), que estabeleceu um plano geral de contas e as respetivas normas de aplicação. A contabilidade de custos assume grande importância no setor público em geral e no SPA em particular, nomeadamente nos municípios, nas áreas da orçamentação, do controlo e redução de custos, da determinação de reembolsos e fixação de taxas e preços públicos, da medição do desempenho na avaliação de programas e na tomada de decisões de tipo económico (FASAB, 1995).

A contabilidade de custos permite conhecer a situação económica e técnica das organizações, o que propicia um controlo de gestão adequado e rigoroso dos recursos públicos; aceder a informação económica de carácter analítico, o que auxilia o processo de planificação e tomada de decisão dos gestores e administradores públicos; e verificar se a organização está a alcançar os objetivos previamente fixados, tomando as necessárias medidas corretivas quando estes não forem atingidos. Estas especificidades assumem um papel importante devido às limitações da informação produzida pelas demonstrações financeiras tradicionais (JOHNSON; KAPLAN, 1987) e à preocupação cada vez mais premente de satisfação de uma grande variedade de necessidades sociais em contínua expansão, atribuídas 
ao setor público em geral e aos municípios em particular, no âmbito das atribuições que lhe são cometidas (Cf. Lei n. ${ }^{\circ}$ 75/2013, de 12 de setembro).

Cardoso, Aquino e Bitti (2011) e Burns e Scapens (2000) sublinham a importância da definição de uma estrutura conceptual (framework) para a informação de custos no setor público, para promover o reforço do nível de accountability e contribuir para a disseminação dos conceitos a outros órgãos da Administração Pública, como uma transferência de conhecimento. Contudo, sublinham que, para que a implementação do sistema de informação de custos se torne uma realidade, não basta a exigência legal nem a definição de um framework, é necessário que os potenciais usuários percebam a relevância da informação que o sistema lhes é capaz de prover, ao que chamam "utilidade percebida da inovação". Importa destacar, que a qualidade da informação dos custos produzidos varia consoante a entidade e o país, o que influencia também a variação do conceito de utilidade (CARDOSO; AQUINO; BITTI, 2011).

Lapsley e Wright (2004) consideram a introdução da contabilidade de gestão, do controlo orçamental e da avaliação de desempenho, as mudanças mais importantes que ocorreram nos últimos anos no âmbito da contabilidade pública.

Para Costa (2005) a contabilidade de custos assume um papel de destaque no setor local, pois disponibiliza informação que permite aferir se os municípios estão a alcançar os objetivos definidos, tomando as medidas corretivas nos casos em que se justifique.

Em Portugal, a contabilidade de custos nos municípios foi impulsionada pela publicação de diferentes diplomas legais, nomeadamente: o Plano Oficial de Contabilidade Pública (POCP), que possibilitou a integração consistente da contabilidade orçamental, patrimonial e de custos numa contabilidade pública moderna; o Plano Oficial de Contabilidade das Autarquias Locais (POCAL), que constitui uma ferramenta fundamental de apoio à gestão das autarquias locais; o Regime Geral de Taxas das Autarquias Locais (RGTAL), que obriga à aplicação do princípio da proporcionalidade na determinação do valor das taxas a praticar pelas autarquias, ou seja, o valor a cobrar ao particular não deve exceder o custo da atividade pública local ou o benefício auferido pelo próprio; a Lei das Finanças Locais (LFL) que destaca a relevância de se aferir o custo da atividade local; e a Lei dos Compromissos e Pagamentos em Atraso (LCPA), que pressupõe que as entidades efetuem um controlo rigoroso e fiável dos custos inerentes aos serviços e às atividades que desenvolvem.

A obrigatoriedade de implementação do SCC está diretamente relacionada com o conceito de utilidade, na medida em que a mesma permite (i) fixar a quantia das taxas e dos preços municipais valorizando de forma proporcional os recursos humanos e materiais que lhe estão subjacentes (RGTAL); e, (ii) aperfeiçoar o processo de orçamentação do setor local, na avaliação das políticas, tendo subjacente uma unidade de medida que permite avaliar as provisões orçamentais e quantificar os custos incorridos no cumprimento dos objetivos definidos nos programas (IGAE, 1994).

Contudo, as reformas que se operaram no seio da NGP, e que tiveram subjacente, entre outros aspetos, a introdução do SCC no Setor Público, não tiveram a mesma configuração e intensidade em todos os países e organizações. As mudanças sentidas são influenciadas, no entender de Hood (1995), pelas especificidades dos países e organizações, nomeadamente, a orientação política, a dimensão e o desempenho macroeconómico, e, no entender de Pollitt e Bouckaert (2004), pelas características do ambiente institucional e cultural no processo de mudança organizacional. Esta influência do ambiente institucional e cultural confronta fatores quer de ordem interna (meios e regras específicos das

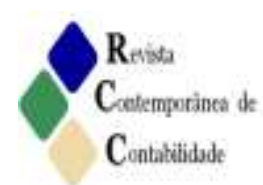


organizações), quer externa (contexto social, sociedade em que a organização está inserida), que podem determinar o próprio processo de mudança interna (BURNS; SCAPENS, 2000).

\section{O Sistema de Contabilidade de Custos Proposto pelo POCAL}

De acordo com o POCAL os municípios devem desenvolver um SCC orientado para as atividades e para os outputs produzidos, o qual visa proporcionar informação adicional à gestão no que respeita à classificação dos custos, por cada função, por cada bem produzido e por cada serviço prestado (Figura 1).

\section{Figura 1 - Sistema de Contabilidade de Custos definido pelo POCAL}

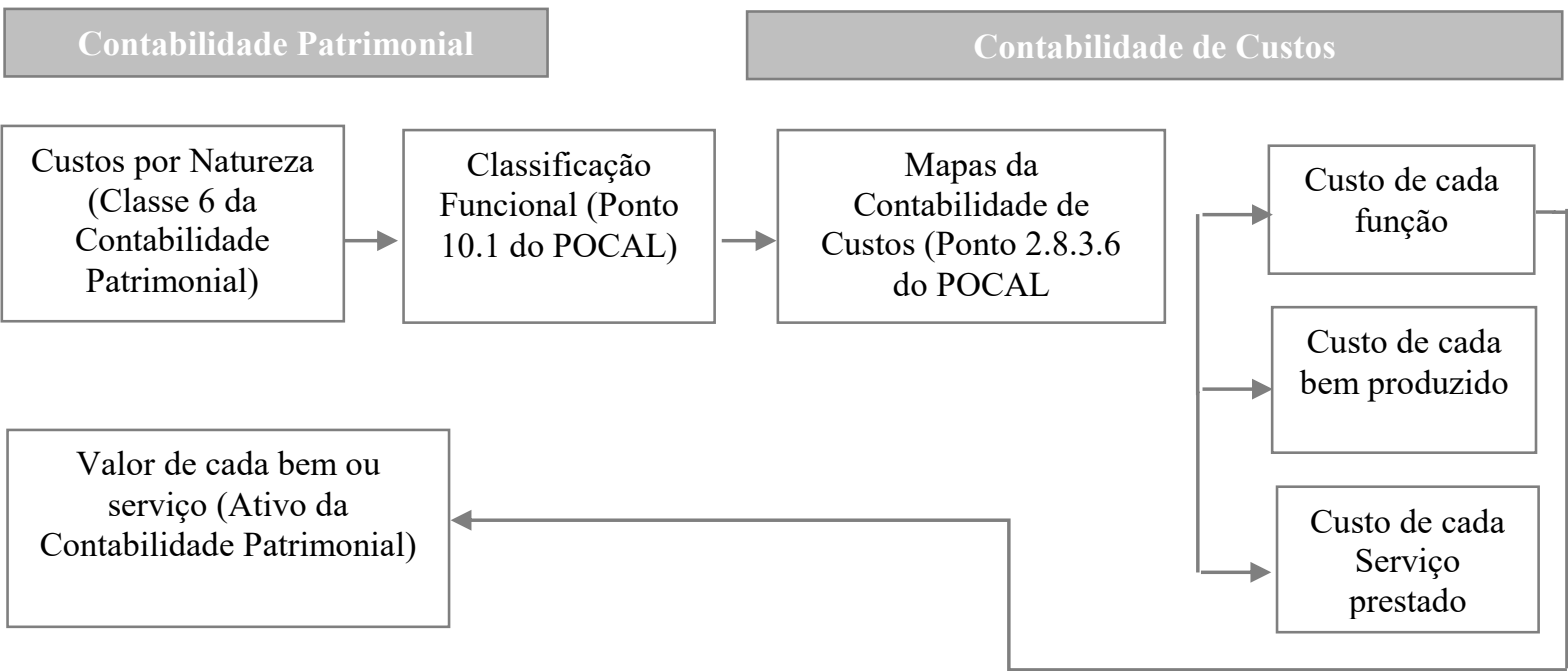

Fonte: SATAPOCAL (2006).

O POCAL não impõe um plano de contas específico para a contabilidade de custos, o que permite às autarquias definirem um plano de contas de acordo com as suas características e complexidade orgânica.

O POCAL prevê a repartição dos custos pelas seguintes rubricas: materiais, mão-deobra, máquinas e viaturas e outros custos diretos; e a reclassificação dos mesmos por natureza, em custos diretos - os que são específica e diretamente suportados por uma função, bem ou serviço e, como tal, exclusivamente imputáveis a esta função, bem ou serviço (COSTA, 2007) e em custos indiretos - os que não podem ser relacionados diretamente com uma função, bem ou serviço, departamento ou atividade, uma vez que não possuem uma relação de causalidade específica entre o input (fator de custo) e o output (COSTA, 2007).

No que respeita à reclassificação dos custos por unidades orgânicas, que tem subjacente o cálculo dos custos de acordo com a estrutura orgânica da entidade, e que na aceção de Santos (2000) se refere a núcleos de recursos humanos e materiais nos quais assenta o desenvolvimento de todo o tipo de projetos da instituição, podendo, de acordo com os seus objetivos específicos, assumir a forma de departamentos ou serviço, o POCAL, não prevê, pelo menos de forma clara, essa reclassificação. No seguimento desta asserção, verificámos que o POCAL não apresenta mapas para o apuramento dos custos das atividades e centros de 
custo, contrariamente ao que acontece em outros planos setoriais (por exemplo no Plano Oficial de Contabilidade do Ministério da Saúde - POCMS) que efetuam o apuramento dos custos através de unidades orgânicas, servindo-se para o efeito e com as devidas adaptações do método das secções homogéneas. Este método, segundo Caiado (2012), refere-se a centros de gastos que respeitam as seguintes características: existência de uma medida comum, unidade de custeio, existência de um só responsável e homogeneidade de funções dentro do mesmo centro de gastos.

Relativamente aos custos indiretos, o POCAL defende que a sua imputação deve ser efetuada através de uma base única de repartição, devendo imputar-se os custos indiretos em função dos custos diretos. A utilização do método da base única apresenta algumas condicionantes, pois só ocasionalmente se encontra uma relação proporcionalmente aceitável entre todos os gastos gerais de fabrico e a base de imputação escolhida (PEREIRA; FRANCO, 1994).

No que respeita ao sistema de custeio, o POCAL não expressa claramente o tipo de sistema a utilizar, no entanto, depreende-se que se deverá optar pelo sistema de custeio total, uma vez que o POCAL considera que devem ser incluídos, no apuramento dos custos os custos diretos e indiretos relacionados com a produção, distribuição, administração geral e financeiros. Este sistema de custeio é no entender de Evans e Bellamy (1995) o sistema que faculta informação mais completa para a tomada de decisão, pois a exclusão de determinados elementos de custo do conceito de custo completo pode condicionar a utilidade da informação deste sistema de custeio.

\section{Metodologia de Investigação}

Os objetivos deste estudo consistem em determinar a fase de implementação do SCC nos municípios portugueses, identificar as determinantes e os constrangimentos da implementação do sistema e o contributo da informação obtida através do mesmo, nomeadamente em termos de utilidade e de accountability para a gestão municipal. Neste sentido foram formuladas as seguintes questões: Quais os fatores impulsionadores e condicionadores da implementação do SCC? Que diferenças existem entre os municípios utilizadores e os não utilizadores do SCC? Quais as características do SCC dos municípios utilizadores? Qual a fase de implementação do SCC? Qual o nível de utilidade retirada da informação produzida pelo SCC a nível interno e externo?

De modo a responder às questões formuladas e tendo em consideração a revisão da literatura efetuada, procurámos testar seis hipóteses: 1) localização geográfica e implementação do SCC; 2) dimensão e implementação do SCC; 3) dimensão e repercussão da informação produzida pelo SCC; 4) dimensão e utilização a nível interno da informação produzida pelo SCC; 5) dimensão e utilização a nível externo da informação produzida pelo SCC; 6) utilização a nível interno da informação produzida pelo SCC e fase de implementação do SCC.

Para tal optámos pela obtenção de dados primários através da aplicação de um inquérito por questionário (técnica quantitativa), dada a escassez de informação documental nesta área. O questionário foi elaborado com base na revisão da literatura, sendo constituído por questões do tipo fechado que se repartiram por questões de resposta única (onde o inquirido seleciona uma opção de uma lista de alternativas pré-selecionada) e por questões

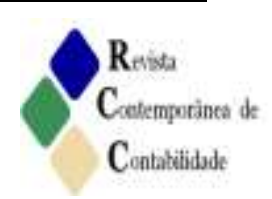


onde se utiliza a escala de Likert (em que o inquirido seleciona, numa escala ordinal entre 0 e 5, o nível de importância, repercussão, utilização e concordância). As questões abarcaram nomeadamente, a caracterização do município, a caracterização do SCC dos municípios utilizadores, a fase de implementação do SCC, a sua repercussão e a sua utilização nos municípios utilizadores, bem como, as condicionantes da implementação do SCC nos municípios utilizadores e não utilizadores.

Após a aplicação do pré-teste do inquérito a cinco municípios, foi enviado por e-mail o link de acesso ao questionário aos 308 municípios portugueses, encontrando-se disponível para resposta entre abril e julho de 2013. O questionário foi dirigido aos Presidentes de Câmara e aos responsáveis da contabilidade. Do total de municípios inquiridos obtivemos 105 respostas que se traduziu numa taxa de resposta de $34 \%$, a qual constitui uma amostra significativa repartida pelas diferentes NUT III, e que permitiu generalizar os resultados obtidos - NUT III é a nomenclatura de Unidade Territorial para fins estatísticos, aprovada pelo Decreto-Lei n. ${ }^{\circ} 46 / 89$, de 15 de fevereiro, que estabeleceu a matriz limitadora da recolha e compilação de informação estatística de base regional em 3 níveis de agregação nível I, II e III.

O tratamento dos dados foi efetuado com recurso ao programa SPSS (Statistical Package for the Social Sciences), versão 20.

No tratamento dos dados considerámos dois tipos de análise: a análise univariada (as variáveis são tratadas de forma independente) e a análise bivariada (as variáveis relacionamse através de cruzamentos). A utilização da análise bivariada teve como finalidade testar as hipóteses formuladas através de medidas de associação:

- H0 (hipótese nula): As variáveis são independentes;

- H1 (hipótese alternativa): As variáveis não são independentes e existe relação entre elas.

As associações baseadas na tabela da contingência foram testadas através de um teste não paramétrico - Teste Exato de Fisher, que avalia a existência de relação entre duas variáveis qualitativas que só podem tomar dois valores cada, considerando-se o nível de significância de 5\%.

\section{Análise dos Resultados}

\subsection{Caracterização dos Inquiridos}

Atendendo às diferentes especificidades dos municípios inquiridos procurámos numa primeira fase proceder à sua caraterização atendendo às seguintes variáveis: localização geográfica, dimensão (número de habitantes e número de trabalhadores) e recursos financeiros (receitas totais e independência financeira).

Em termos de localização geográfica, caracterizámos os municípios inquiridos, por NUT III, verificando-se no Continente uma maior taxa de resposta $(>75 \%)$ no Médio Tejo e no Pinhal Litoral. Contrariamente, as regiões com menor taxa de resposta $(\leq 25 \%)$ foram o Minho Lima, o Alto Trás-os-Montes, Baixo Vouga, Beira Interior Norte, Beira Interior Sul, Cova da Beira, Alentejo Litoral, Alto Alentejo e Alentejo Central. 
No que concerne às Regiões Autónomas verificámos que as taxas de resposta foram mais baixas do que no Continente. Nos Açores a taxa de resposta cifrou-se nos $16 \%$ e na Madeira não foi além dos $9 \%$.

A dimensão dos inquiridos foi analisada de acordo com os critérios do número de habitantes e do número de trabalhadores dos municípios (Tabela 1). No que respeita ao número de habitantes, utilizámos a escala definida no Anuário Financeiro dos Municípios Portugueses (Carvalho et al., 2013), na qual os municípios se enquadram em três grupos: pequenos ( $\leq 20.000$ habitantes), médios (entre 20.001 a 100.000 habitantes) e grandes $(\geq 100.001$ habitantes).

Relativamente ao número de trabalhadores, agrupámos os municípios em três grupos: pequenos ( $\leq 300$ trabalhadores), médios (entre 301 a 600 trabalhadores) e grandes $(\geq 601$ trabalhadores).

Tabela 1 - Distribuição dos municípios inquiridos em função do critério do n. ${ }^{0}$ de habitantes e do n. ${ }^{0}$ de trabalhadores, dados de julho de 2013

\begin{tabular}{|l|r|r|r|r|r|}
\multicolumn{1}{c|}{ Dimensão } & \multicolumn{2}{|c|}{ Número de Habitantes } & \multicolumn{3}{c|}{ Número de Trabalhadores } \\
\hline Pequena Dimensão & N & \multicolumn{1}{c|}{$\%$} & N & $\%$ \\
\hline Média Dimensão & 56 & $53 \%$ & 59 & $56 \%$ \\
\hline Grande Dimensão & 37 & $35 \%$ & 24 & $23 \%$ \\
\hline Total & 12 & $12 \%$ & 22 & $21 \%$ \\
\hline
\end{tabular}

Fonte: Elaborado pelos autores.

Da análise dos dados verificámos que o grupo de municípios inquiridos com maior peso em termos de número de habitantes é o grupo de pequena dimensão que representa $53 \%$ (56 municípios). Contrariamente, o grupo com menor peso é o grupo de grande dimensão que representa 12\% (12 municípios). Esta análise é extensiva ao critério do número de trabalhadores, sendo o grupo com maior peso o de pequena dimensão, o qual representa $56 \%$ (59 municípios). Inversamente, o grupo com menor peso é o de grande dimensão que representa $21 \%$ (22 municípios).

No que respeita aos recursos financeiros, caracterizámos os municípios de acordo com dois critérios: receitas totais e independência financeira.

Em termos de volume de receitas definimos um intervalo de forma a agruparmos os municípios em quatro grupos: $\leq 10.000 .000 €$; entre 10.000.001€ e 20.000.000€; entre 20.000.001€ e 30.000.00€; e $\geq 30.000 .001 €$. De acordo com o intervalo apresentado, constatámos que a maior parte dos municípios inquiridos apresenta um volume de receitas entre $10.000 .001 €$ e $20.000 .000 €$, o que corresponde a $36 \%$ (38 municípios) e apenas $29 \%$ dos municípios inquiridos apresentam um volume de receitas superior a 30.000.001€ (29 municípios).

Relativamente à independência financeira dos municípios (quando as receitas próprias - receitas totais deduzidas das transferências e passivos financeiros, representam pelo menos $50 \%$ das receitas totais), verificámos que no ano de $2011,78 \%$ dos municípios (81 municípios) não são independentes financeiramente, o que significa que as transferências (correntes e de capital) representam mais de $50 \%$ do total das receitas, evidenciando a forte dependência financeira dos municípios inquiridos das transferências da Administração 
Central. A dependência demonstrada não se verifica apenas nos municípios inquiridos dado que, de acordo com o Anuário Financeiro dos Municípios Portugueses (Carvalho et al., 2013), em 2011, apenas 33\% do total dos municípios portugueses (101 municípios) apresentavam receitas próprias iguais ou superiores a $50 \%$ das receitas totais.

\subsection{Apresentação e Discussão dos Resultados}

No que respeita à implementação do SCC, verificámos que 58\% dos municípios (61 municípios) já implementou este sistema. Importa referir que, apesar do resultado obtido ainda se considerar reduzido face à obrigatoriedade legal, se registou um aumento relativamente ao estudo de Gomes et al. (2009), no qual a percentagem de implementação era de $48 \%$.

Em termos da fase de implementação do SCC, verificámos que $41 \%$ dos municípios que já implementaram o SCC (25 municípios) considerou encontrar-se numa fase intermédia de implementação, sendo possível extrair informação do SCC para utilização a nível interno ou externo. De salientar que apenas 3\% (2 municípios) considerou encontrar-se numa fase embrionária, da qual, ainda não é possível retirar informação relevante do SCC.

Em matéria de regras de operacionalização do SCC, verificámos que não existe uniformização entre os municípios utilizadores. A norma mais comum é o apuramento dos custos através de centro de custos, que corresponde a 44\% dos municípios (apesar do POCAL estabelecer que o cálculo dos custos deve recair sobre os bens e serviços após a delimitação prévia dos mesmos). De referir que, apesar da forma de apuramento dos custos mais utilizada ser a dos centros de custos, nem todos os municípios cumprem estritamente todas as regras inerentes a esta forma de apuramento, dado que a mesma pressupõe a utilização do método das secções homogéneas e apenas $28 \%$ dos municípios confirmaram a aplicação deste método (o que corresponde a 59\% dos municípios que utilizam o método dos centros de custos). O tipo de sistema de custeio mais utilizado é o preconizado implicitamente pelo POCAL - o sistema de custeio total, que é aplicado por $93 \%$ dos municípios. A utilização deste sistema pela maioria dos municípios deve-se ao facto de este permitir o fornecimento de informação sobre o custo completo dos bens e serviços, dado considerar todos os custos (fixos e variáveis), o que faculta aos administradores e gestores uma fonte de informação completa que apoia de forma mais rigorosa o processo de tomada de decisão. Em relação aos custos indiretos, constatámos que $74 \%$ dos municípios fazem a sua imputação, sendo a forma de imputação mais utilizada a de base única, correspondendo a 53\% dos municípios. Este método consiste na imputação dos custos indiretos em função dos custos diretos, através de uma única base de imputação. Refere-se, no entanto, que esta base de imputação tem um elevado grau de subjetividade, dada a dificuldade em encontrar uma relação de proporcionalidade aceitável entre os custos a repartir e a base de imputação escolhida.

De acordo com a tabela 2, verificámos que a implementação do SCC foi mais acentuada a partir do ano de 2006. Neste sentido confirma-se que os diplomas com maior impacto na implementação do SCC, foram o RGTAL (Lei n. ${ }^{\circ}$ 53-E/2006, de 29 de dezembro) e a LFL (Lei.$^{\circ}{ }^{2} 2007$, de 15 de janeiro), uma vez que estes diplomas entraram em vigor no ano de 2007 e tinham subjacente, no caso do RGTAL, justificar económica e financeiramente as taxas praticadas pelos municípios nos bens e serviços prestados, e, no caso da LFL, aumentar a transparência das finanças autárquicas. Além disso, importa salientar que nenhum 
dos municípios implementou o SCC antes de 1999, ano em que foi aprovado o POCAL, este facto reforça a influência que a legislação tem na implementação do SCC.

No que respeita aos fatores impulsionadores, constatámos de acordo com a tabela que, na opinião dos utilizadores, o fator com maior contributo para a implementação do SCC foi a obrigatoriedade imposta pela legislação (POCP, POCAL, RGTAL e LFL), obtendo uma média de 4,18 (escala 0 a 5). A importância deste fator é também confirmada pelo ano de implementação do SCC, dado que nenhum dos municípios inquiridos implementou o SCC antes da data de aprovação do POCP e do POCAL. No mesmo sentido, Lapsley e Wright (2004) concluem que 67\% das autarquias locais na Escócia (Local Authorities) admitem que as técnicas contabilísticas são introduzidas por imposição legal e apenas $28 \%$ para dar resposta a necessidades de informação. Quanto à publicação de legislação, destacamos a contribuição do RGTAL e da LFL, uma vez que a implementação do SCC passou a ser mais significativa a partir do ano de 2006, após a entrada em vigor destes diplomas. Este resultado não difere substancialmente do obtido por Gomes et al. (2009), que referem o POCAL e o RGTAL como os diplomas com maior contribuição na implementação do SCC, verificandose que nenhum dos municípios implementou o SCC antes da entrada em vigor do POCAL e que a grande maioria apenas implementou o SCC após a aprovação do RGTAL e da LFL. Contrariamente, o fator com menor influência na opinião dos utilizadores, foi a pressão política, o qual obteve uma média de 2,56.

No que concerne à repercussão da informação produzida pelo SCC na melhoria da gestão do município em termos de economia, eficiência e eficácia, verificámos uma contribuição ligeira para a melhoria da gestão do município, uma vez que a média das respostas se situou nos 3,20. De acordo com o estudo realizado, esta situação poderá estar relacionada com o facto de a maior parte dos municípios utilizadores se encontrar numa fase intermédia de implementação do SCC, que não permite ainda possuir um conhecimento adequado da informação que é possível retirar do SCC.

Relativamente à utilização da informação produzida pelo SCC, constatámos que é utilizada de forma moderada, quer a nível interno (média: 3,28), quer a nível externo (média: 3,02). Tal como na questão anterior, esta situação poderá ser explicada pela fase de implementação do SCC em que se encontra a maior parte dos municípios utilizadores.

Os fatores condicionadores da implementação do SCC foram analisados separadamente para os municípios utilizadores e para os municípios não utilizadores, constatando-se que todos os fatores estudados evidenciam na generalidade uma importância razoável para as duas categorias de municípios. No entanto, os fatores que mereceram maior importância não são comuns entre os municípios utilizadores e os não utilizadores, uma vez que, para os municípios utilizadores, o principal obstáculo à implementação do SCC foi a resistência por parte dos funcionários públicos ao controlo inerente ao SCC (média: 3,79) e, para os municípios não utilizadores, o principal obstáculo foi a escassez de recursos humanos (média: 3,86).

No mesmo sentido, num estudo realizado por Carvalho, Costa e Macedo (2008) foram identificados alguns constrangimentos à implementação do SCC, como a não exigibilidade de entrega dos documentos gerados pelo SCC ao Tribunal de Contas, as dificuldades na escolha de bases de repartição dos custos indiretos, a escassez de recursos humanos, a minimização do papel da contabilidade analítica comparativamente com a contabilidade orçamental e patrimonial, a falta de tempo e a falta de vontade política. 
Tabela 2 - Estatísticas Descritivas

\begin{tabular}{|c|c|c|c|c|c|c|c|}
\hline \multicolumn{3}{|c|}{ Estatísticas Descritivas } & $\mathbf{N}$ & Média & Desvio & Mínimo & Máximo \\
\hline \multicolumn{3}{|c|}{$\begin{array}{l}\text { Ano de Implementação do SCC (1 - Entre } 2001 \text { e } 2005 \text { / } 2 \text { - Entre } 2006 \text { e } 2011 \\
\text { / 3-2012) }\end{array}$} & 61 & 2,08 & 0,493 & 1 & 3 \\
\hline $\begin{array}{l}\text { Fatores } \\
\text { Impulsionadores }\end{array}$ & \multicolumn{2}{|c|}{$\begin{array}{l}\text { Obrigatoriedade Imposta pela Legislação (POCP, } \\
\text { POCAL, RGTAL, LFL }\end{array}$} & 61 & 4,18 & 0,785 & 2 & 5 \\
\hline $\begin{array}{l}\text { da Implementação } \\
\text { do SCC }\end{array}$ & \multicolumn{2}{|c|}{$\begin{array}{l}\text { Necessidade de Aumentar a Transparência das Finanças } \\
\text { Autárquicas }\end{array}$} & 61 & 3,61 & 0,954 & 1 & 5 \\
\hline $\begin{array}{l}\text { (1 - Nenhuma } \\
\text { Importância / } \\
\text { 2- Pouca }\end{array}$ & \multicolumn{2}{|c|}{$\begin{array}{l}\text { Necessidade de Controlar os Custos decorrentes dos } \\
\text { Atuais Constrangimentos Orçamentais Impostos pela } \\
\text { LCPA }\end{array}$} & 60 & 3,67 & 1,036 & 1 & 5 \\
\hline $\begin{array}{c}\text { Importância / } \\
\text { 3 - Importância } \\
\text { Razoável / } \\
\text { 4 - Importância } \\
\text { Elevada / } \\
5 \text { - Importância } \\
\text { Muito Elevada) }\end{array}$ & \multicolumn{2}{|c|}{ Pressão Política } & 59 & 2,56 & 1,087 & 1 & 5 \\
\hline \multicolumn{3}{|c|}{$\begin{array}{l}\text { Repercussão da Informação produzida pelo SCC na melhoria da Gestão d } \\
\text { Município em termos de Economia, Eficiência e Eficácia (1 - Nenhuma } \\
\text { Repercussão / } 2 \text { Pouca Repercussão / } 3 \text { - Repercussão Razoável / } 4 \text { - } \\
\text { Repercussão Elevada / } 5 \text { - Repercussão Muito Elevada) }\end{array}$} & 61 & 3,20 & 0,792 & 1 & 5 \\
\hline \multicolumn{3}{|c|}{$\begin{array}{l}\text { Utilização da Informação produzida pelo SCC a nível Interno (1 - Nenhuma } \\
\text { Utilização / } 2 \text { Pouca Utilização / } 3 \text { - Utilização Razoável / } 4 \text { - Utilização } \\
\text { Elevada / } 5 \text { - Utilização Muito Elevada) }\end{array}$} & 61 & 3,28 & 0,819 & 1 & 5 \\
\hline \multicolumn{3}{|c|}{$\begin{array}{l}\text { Utilização da Informação produzida pelo SCC } \\
\text { Utilização / } 2 \text { Pouca Utilização / } 3 \text { - Utilização } \\
\text { Elevada / } 5 \text { - Utilização Muito Elevada) }\end{array}$} & 61 & 3,02 & 0,904 & 1 & 5 \\
\hline \multirow{5}{*}{$\begin{array}{c}\text { Fatores } \\
\text { Condicionadores } \\
\text { da Implementação } \\
\text { do SCC (1 - } \\
\text { Nenhuma }\end{array}$} & \multirow{5}{*}{$\begin{array}{l}\text { Municípios } \\
\text { Adotantes }\end{array}$} & Escassez de Recursos Humanos & 61 & 3,70 & 0,843 & 1 & 5 \\
\hline & & Escassez de Recursos Financeiros & 61 & 2,82 & 0,806 & 1 & 5 \\
\hline & & Escassez de Meios Técnicos & 61 & 3,56 & 1.041 & 1 & 5 \\
\hline & & $\begin{array}{l}\text { Escassez de de Formação Adequada sobre } \\
\text { o SCC }\end{array}$ & 61 & 3,64 & 0,949 & 2 & 5 \\
\hline & & $\begin{array}{l}\text { Escassez de Mecanismos de Fiscalização da } \\
\text { sua Implementação }\end{array}$ & 61 & 3,74 & 0,835 & 2 & 5 \\
\hline
\end{tabular}




\begin{tabular}{|c|c|c|c|c|c|c|c|}
\hline $\begin{array}{c}\text { Importância / } 3 \text { - } \\
\text { Importância }\end{array}$ & & $\begin{array}{l}\text { Dificuldades de interpretação das Regras } \\
\text { do POCAL }\end{array}$ & 61 & 3,03 & 0,856 & 1 & 5 \\
\hline Razoável / 4 - & & Resistência à Mudança & 61 & 3,75 & 1,090 & 1 & 5 \\
\hline $\begin{array}{l}\text { Importância } \\
\text { Elevada / } 5 \text { - }\end{array}$ & & $\begin{array}{l}\text { Resistência ao Controlo Subjacente ao } \\
\text { SCC }\end{array}$ & 61 & 3,79 & 1,199 & 1 & 5 \\
\hline \multirow[t]{10}{*}{$\begin{array}{l}\text { Mmportancia } \\
\text { Muito Elevada) }\end{array}$} & & $\begin{array}{l}\text { Dificuldades de Recolha e Interpretação } \\
\text { dos Dados - SCC }\end{array}$ & 61 & 3,64 & 0,913 & 1 & 5 \\
\hline & \multirow{9}{*}{$\begin{array}{l}\text { Municípios } \\
\text { não } \\
\text { Adotantes }\end{array}$} & Escassez de Recursos Humanos & 44 & 3,86 & 1,193 & 1 & 5 \\
\hline & & Escassez de Recursos Financeiros & 44 & 2,98 & 1,229 & 1 & 5 \\
\hline & & Escassez de Meios Técnicos & 44 & 3,27 & 1,208 & 1 & 5 \\
\hline & & $\begin{array}{l}\text { Escassez de Formação Adequada sobre o } \\
\text { SCC }\end{array}$ & 44 & 3,27 & 1,065 & 1 & 5 \\
\hline & & $\begin{array}{l}\text { Escassez de Mecanismos de Fiscalização da } \\
\text { sua Implementação }\end{array}$ & 44 & 3,11 & 1,083 & 1 & 5 \\
\hline & & $\begin{array}{l}\text { Dificuldades de interpretação das Regras } \\
\text { do POCAL }\end{array}$ & 44 & 2,36 & 1,080 & 1 & 5 \\
\hline & & Resistência à Mudança & 44 & 2,82 & 1,352 & 1 & 5 \\
\hline & & $\begin{array}{l}\text { Resistência por ao Controlo Subjacente ao } \\
\text { SCC }\end{array}$ & 44 & 2,73 & 1,264 & 1 & 5 \\
\hline & & $\begin{array}{l}\text { Dificuldades de Recolha e Interpretação } \\
\text { dos Dados - SCC }\end{array}$ & 44 & 3,18 & 1,225 & 1 & 5 \\
\hline
\end{tabular}

Fonte: Elaborado pelos autores.

\subsection{Análise das Hipóteses}

Na hipótese 1 pretendeu-se testar a associação entre a localização geográfica e a implementação do SCC, uma vez que são as regiões do litoral que registam maiores densidades populacionais, destacando-se a faixa do litoral entre o Minho-Lima e a Península de Setúbal. Além disso, é nas regiões do litoral que se localizam o maior número de municípios e instituições de ensino superior, as quais estão mais dispostas a ministrar disciplinas de contabilidade pública e de contabilidade analítica (COSTA, 2007).

$\mathrm{Na}$ análise dos dados verificámos que o nível de significância no Teste Exato de Fisher é de 0,199 (p-value), logo não podemos rejeitar a hipótese nula (H0) que afirma que as variáveis localização geográfica e implementação do SCC são independentes, não se verificando assim associação.

A implementação do SCC é mais relevante nas NUT III que não integram a faixa do litoral, representando $61 \%$ dos municípios (Tabela 3). Esta realidade é explicada pelo facto das diferenças entre o litoral e o interior estarem atualmente mais esbatidas e por existir uma preocupação cada vez mais acentuada por parte dos municípios do interior em adotar os procedimentos estabelecidos no âmbito do POCAL. 
Tabela 3 - Localização geográfica (NUT III) e Implementação do SCC, dados de julho de 2013

\begin{tabular}{|c|c|c|c|c|c|}
\hline \multirow{2}{*}{\multicolumn{3}{|c|}{ NUT III: Litoral vs Restantes NUT III }} & \multicolumn{2}{|c|}{ Implementação do SCC } & \multirow{2}{*}{ Total } \\
\hline & & & Sim & Não & \\
\hline \multirow{4}{*}{ Litoral } & \multirow{2}{*}{ Sim } & Casos Observados & 15 & 15 & 30 \\
\hline & & \% NUT III: Litoral & $50 \%$ & $50 \%$ & $100 \%$ \\
\hline & \multirow{2}{*}{ Não } & Casos Observados & 46 & 29 & 75 \\
\hline & & \% Restantes NUT III & $61 \%$ & $39 \%$ & $100 \%$ \\
\hline \multirow{2}{*}{\multicolumn{2}{|c|}{ Total }} & Casos Observados & 61 & 44 & 105 \\
\hline & & $\begin{array}{l}\text { \% Total de Casos } \\
\text { Observados }\end{array}$ & $58 \%$ & $42 \%$ & $100 \%$ \\
\hline
\end{tabular}

Fonte: Elaborado pelos autores.

Na hipótese 2 pretendeu-se testar a associação entre a dimensão e a implementação do SCC, ou seja, apreender se os municípios com maior dimensão em termos de número de habitantes e número de trabalhadores apresentam maior propensão à implementação do SCC, uma vez que este sistema possibilita o controlo mais rigoroso dos custos do município.

Considerámos municípios de grande dimensão, os municípios com um número de habitantes $>=100.001$ habitantes e com um número de trabalhadores $>=601$ trabalhadores.

De acordo com o Teste Exato de Fisher, verificámos que o nível de significância obtido, é de 0,619 ( $p$-value), logo não existe evidência estatística que permita rejeitar a hipótese nula (H0), que afirma que as variáveis dimensão e implementação do SCC são independentes, não sendo possível verificar associação entre estas variáveis.

As taxas de implementação do SCC nos municípios de grande dimensão e nos municípios de pequena e média dimensão apresentam percentagens semelhantes, verificandose que, contrariamente ao que poderia ser expectável, a variável dimensão não é condição essencial para a implementação do SCC (Tabela 4). Esta propensão à implementação do SCC nos municípios de pequena e média dimensão pode ser explicada pela homogeneidade da cultura destes municípios e pela maior capacidade de adaptação à mudança tal como avançado anteriormente por Gomes et al. (2009). Neste sentido conclui-se que a complexidade organizacional inerente aos municípios de grande dimensão pode condicionar a implementação do SCC. No mesmo sentido, Carvalho, Jorge e Fernandes (2007) concluíram que a dimensão da organização tem um efeito negativo na implementação do SCC, devido à complexidade organizacional, às práticas conservadoras e à aversão à mudança, por oposição à condição financeira que tem um efeito positivo.

Tabela 4 - Dimensão e Implementação do SCC, dados de julho de 2013

\begin{tabular}{|c|c|c|}
\hline \multicolumn{3}{|c|}{$\begin{array}{c}\text { Municípios de Grande Dimensão vs Municípios de Pequena } \\
\text { e Média Dimensão }\end{array}$} \\
\hline \multirow{4}{*}{$\begin{array}{l}\text { Grande } \\
\text { Dimensão }\end{array}$} & \multirow{2}{*}{ Sim } & Casos Observados \\
\hline & & \% Municípios de Grande Dimensão \\
\hline & \multirow[b]{2}{*}{ Não } & Casos Observados \\
\hline & & $\begin{array}{l}\text { \% Municípios de Pequena e Média } \\
\text { Dimensão }\end{array}$ \\
\hline \multirow{2}{*}{\multicolumn{2}{|c|}{ Total }} & Casos Observados \\
\hline & & $\%$ Total de Casos Observados \\
\hline
\end{tabular}

\begin{tabular}{|r|r|r|}
\hline \multicolumn{1}{|c|}{ Implementação do SCC } & \multicolumn{2}{c|}{ Total } \\
\hline \multicolumn{1}{|c|}{ Sim } & \multicolumn{1}{|c|}{ Não } & 12 \\
\hline 7 & 5 & $100 \%$ \\
\hline 54 & $42 \%$ & 93 \\
\hline $58 \%$ & 49 & $100 \%$ \\
\hline 61 & 44 & 105 \\
\hline $58 \%$ & $42 \%$ & $100 \%$ \\
\hline
\end{tabular}

Fonte: Elaborado pelos autores. 
Na hipótese 3 pretendeu-se testar a associação entre a dimensão e a repercussão da informação produzida pelo SCC na gestão municipal, de forma a percebermos se os outputs produzidos pelo SCC se repercutem de forma significativa na gestão municipal e se, por sua vez, esta repercussão é mais notória nos municípios de maior dimensão. Assim, considerou-se como uma repercussão acentuada a que se classifica como "elevada" e "muito elevada".

Após a aplicação do Teste Exato de Fisher obtivemos um nível de significância de 0,624 ( $p$-value) não se podendo deste modo rejeitar a hipótese nula (H0), que afirma que as variáveis dimensão e repercussão da informação produzida pelo SCC são independentes.

Em termos de repercussão do SCC na melhoria da gestão, verificamos que apenas $29 \%$ dos municípios de grande dimensão e $32 \%$ dos municípios de pequena e média dimensão consideram que este sistema se repercute de forma acentuada na gestão do município (Tabela 5). Esta repercussão é, em termos globais, reduzida, representando $31 \%$ dos casos observados, o que pode ser explicado pelo desconhecimento dos funcionários dos municípios sobre os contributos deste sistema para a melhoria da gestão. No estudo realizado os utilizadores apontaram como principais fatores condicionadores à implementação do SCC a resistência por parte dos funcionários, à mudança e ao controlo inerente ao SCC.

Tabela 5 - Dimensão e Repercussão da informação produzida pelo SCC, dados de julho de 2013

\begin{tabular}{|c|c|c|c|c|c|}
\hline \multirow{2}{*}{\multicolumn{3}{|c|}{$\begin{array}{c}\text { Municípios de Grande Dimensão vs Municípios de Pequena } \\
\text { e Média Dimensão }\end{array}$}} & \multicolumn{2}{|c|}{$\begin{array}{l}\text { Repercussão acentuada } \\
\text { da Informação do SCC }\end{array}$} & \multirow[t]{2}{*}{ Total } \\
\hline & & & Sim & Não & \\
\hline \multirow{4}{*}{$\begin{array}{l}\text { Grande } \\
\text { Dimensão }\end{array}$} & \multirow{2}{*}{ Sim } & Casos Observados & 2 & 5 & 7 \\
\hline & & \% Municípios de Grande Dimensão & $29 \%$ & $71 \%$ & $100 \%$ \\
\hline & \multirow[b]{2}{*}{ Não } & Casos Observados & 17 & 37 & 54 \\
\hline & & $\begin{array}{l}\text { \% Municípios de Pequena e Média } \\
\text { Dimensão }\end{array}$ & $32 \%$ & $68 \%$ & $100 \%$ \\
\hline \multirow{2}{*}{\multicolumn{2}{|c|}{ Total }} & Casos Observados & 19 & 42 & 61 \\
\hline & & $\%$ Total de Casos Observados & $31 \%$ & $69 \%$ & $100 \%$ \\
\hline
\end{tabular}

Fonte: Elaborado pelos autores.

Na hipótese 4 pretendeu-se testar a associação entre a dimensão e a utilização da informação produzida pelo SCC a nível interno, de modo a aferirmos a utilidade da informação produzida pelo SCC (na ótica da utilização da informação), por exemplo, ao nível da elaboração dos documentos previsionais. Deste modo, consideramos como utilização acentuada a nível interno uma utilização "elevada" e "muito elevada".

Da análise dos dados constatamos que o nível de significância obtido após a aplicação do Teste Exato de Fisher é de 0,427 (p-value), pelo que, não podemos rejeitar a hipótese nula (H0), que afirma que as variáveis dimensão e utilização da informação produzida pelo SCC a nível interno são independentes.

Em termos de utilização da informação produzida pelo SCC a nível interno, verificámos que, dos municípios de grande dimensão, apenas 29\% dos municípios utilizam de forma elevada a informação produzida por este sistema, nomeadamente ao nível de elaboração dos documentos previsionais, ao nível da elaboração da prestação de contas e ao nível da tomada de decisão. Nos municípios de pequena e média dimensão, a utilização da informação 
produzida por este sistema tem uma maior aceitação, representando $41 \%$ dos municípios, que se distribuem por uma utilização elevada e muito elevada. Este resultado está em consonância com a verificação anterior, confirmando-se uma utilização mais acentuada a nível interno nos municípios de pequena e média dimensão, apesar de, em termos globais, a utilização representar apenas 39\% dos municípios (Tabela 6).

Tabela 6 - Dimensão e Utilização da informação produzida pelo SCC a nível interno, dados de julho de 2013

\begin{tabular}{|c|c|c|c|c|c|}
\hline \multirow{2}{*}{\multicolumn{3}{|c|}{$\begin{array}{l}\text { Municípios de Grande Dimensão vs Municípios de Pequena } \\
\text { e Média Dimensão }\end{array}$}} & \multicolumn{2}{|c|}{$\begin{array}{l}\text { Utilização acentuada a nível } \\
\text { interno da Informação do SCC }\end{array}$} & \multirow[t]{2}{*}{ Total } \\
\hline & & & Sim & Não & \\
\hline \multirow{4}{*}{$\begin{array}{l}\text { Grande } \\
\text { Dimensão }\end{array}$} & \multirow{2}{*}{ Sim } & Casos Observados & 2 & 5 & 7 \\
\hline & & \% Municípios de Grande Dimensão & $29 \%$ & $71 \%$ & $100 \%$ \\
\hline & \multirow[b]{2}{*}{ Não } & Casos Observados & 22 & 32 & 54 \\
\hline & & $\begin{array}{l}\text { \% Municípios de Pequena e Média } \\
\text { Dimensão }\end{array}$ & $41 \%$ & $59 \%$ & $100 \%$ \\
\hline \multirow{2}{*}{ Total } & & Casos Observados & 24 & 37 & 61 \\
\hline & & $\%$ Total de Casos Observados & $39 \%$ & $61 \%$ & $100 \%$ \\
\hline
\end{tabular}

Fonte: Elaborado pelos autores.

$\mathrm{Na}$ hipótese 5 pretendeu-se testar a associação entre a dimensão e a utilização da informação produzida pelo SCC a nível externo, de modo a aferirmos a utilidade da informação produzida pelo SCC (na ótica da utilização) no reporte de informação a entidades externas. Considerámos como uma utilização acentuada a nível externo, uma utilização "elevada" e "muito elevada".

De acordo com a aplicação do Teste Exato de Fisher verificamos que o nível de significância obtido foi de 0,325 (p-value). Deste modo constatamos que as variáveis dimensão e utilização a nível externo da informação produzida pelo SCC são independentes, tal como o verificado na hipótese anterior relativamente à utilização a nível interno.

Em termos de utilização da informação produzida pelo SCC a nível externo, verificámos que apenas $14 \%$ dos municípios de grande dimensão utilizam de forma significativa a informação produzida pelo SCC, nomeadamente ao nível de reporte de informação a outras entidades (exemplo: DGAL, INE). 32\% dos municípios de pequena e média dimensão repartiram-se pela utilização elevada e muito elevada, pelo que podemos concluir que a utilização a nível externo da informação produzida pelo SCC é mais acentuada nos municípios de pequena e média dimensão, apesar de, em termos globais, ser ainda pouco utilizada, representando apenas $30 \%$ dos municípios (Tabela 7).

$\mathrm{Na}$ hipótese 6 pretendeu-se testar a associação entre a utilização da informação produzida pelo SCC a nível interno e a fase de implementação do SCC, de modo a percebermos se a utilização dos outputs produzidos pelo SCC está relacionada com a fase em que se encontra a implementação do SCC, ou seja, se os municípios que se encontram mais avançados em termos de implementação do SCC são os que utilizam mais a informação produzida pelo SCC. Para tal, selecionámos os municípios onde o SCC se encontra numa fase "avançada" e "totalmente implementada", de forma a destacá-los dos restantes municípios que se encontram ainda numa fase "inicial" e "intermédia". 
Tabela 7 - Dimensão e Utilização da informação produzida pelo SCC a nível externo, dados de julho de 2013

\begin{tabular}{|c|c|c|c|c|c|}
\hline \multirow{2}{*}{\multicolumn{3}{|c|}{$\begin{array}{c}\text { Municípios de Grande Dimensão vs Municípios de Pequena } \\
\text { e Média Dimensão }\end{array}$}} & \multicolumn{2}{|c|}{$\begin{array}{l}\text { Utilização acentuada a nível } \\
\text { externo da Informação do } \\
\text { SCC }\end{array}$} & \multirow[t]{2}{*}{ Total } \\
\hline & & & Sim & Não & \\
\hline \multirow{4}{*}{$\begin{array}{c}\text { Grande } \\
\text { Dimensão }\end{array}$} & \multirow{2}{*}{ Sim } & Casos Observados & 1 & 6 & \\
\hline & & \% Municípios de Grande Dimensão & $14 \%$ & $86 \%$ & $100 \%$ \\
\hline & \multirow[b]{2}{*}{ Não } & Casos Observados & 17 & 37 & 54 \\
\hline & & $\begin{array}{l}\text { \% Municípios de Pequena e Média } \\
\text { Dimensão }\end{array}$ & $32 \%$ & $86 \%$ & $100 \%$ \\
\hline \multirow{2}{*}{\multicolumn{2}{|c|}{ Total }} & Casos Observados & 18 & 43 & 61 \\
\hline & & $\%$ Total de Casos Observados & $29 \%$ & $71 \%$ & $100 \%$ \\
\hline
\end{tabular}

Fonte: Elaborado pelos autores.

Após a aplicação do Teste Exato de Fisher obtivemos um nível de significância de 0,131 (p-value), o que não nos permite rejeitar a hipótese nula que afirma que as variáveis são independentes.

Deste modo, constatamos que a fase em que se encontra a implementação do SCC não influencia, pelo menos diretamente, a utilização dos outputs produzidos pelo SCC. Dos 34 municípios que consideraram que o SCC estava numa fase de implementação "avançada" ou "totalmente implementada", apenas 16 municípios consideraram utilizar de forma permanente a informação produzida pelo SCC (Tabela 8). Neste sentido, verificou-se que as proposições avançadas, no sentido de que os baixos níveis de repercussão do SCC na gestão do município e de utilização da informação produzida pelo SCC, poderiam estar relacionadas com a fase de implementação, não ficaram comprovadas.

Do estudo efetuado, constata-se que a utilização pouco relevante dos outputs produzidos pelo SCC pode estar relacionada com o facto de o SCC ser um instrumento inovador e complexo, o que dificulta a interpretação dos seus contributos para a melhoria da gestão municipal, tomada de decisão, racionalização dos recursos públicos e promoção da accountability.

Tabela 8 - Utilização da informação produzida pelo SCC a nível interno e Fase de implementação do SCC, dados de julho de 2013

\begin{tabular}{|c|l|}
\hline Utilização acentuada a nível interno da informação produzida \\
pelo SCC
\end{tabular}

\begin{tabular}{|r|r|r|}
$\begin{array}{r}\text { Fase de Implementação do } \\
\text { SCC Avançada e Totalmente } \\
\text { Implementada }\end{array}$ & \multicolumn{2}{l}{ Total } \\
\hline Sim & \multicolumn{2}{|c|}{ Não } \\
\hline 16 & 8 & 24 \\
\hline $67 \%$ & $33 \%$ & $100 \%$ \\
\hline 18 & 19 & 37 \\
\hline $39 \%$ & $51 \%$ & $100 \%$ \\
\hline $56 \%$ & 27 & 61 \\
\hline
\end{tabular}

Fonte: Elaborado pelos autores 


\section{Conclusão}

O POCAL determinou a obrigatoriedade de implementação do SCC nos municípios portugueses e estabeleceu um conjunto de regras para efetivar o seu desenvolvimento, contudo ainda há muitos municípios que não aplicam esta técnica.

A introdução do SCC teve como finalidade colmatar as insuficiências de informação das demonstrações financeiras tradicionais, disponibilizando assim informação muito específica para a tomada de decisão e o controlo rigoroso e pertinente dos custos, prosseguindo objetivos de economia, de eficiência e de eficácia nos serviços autárquicos.

Os resultados deste estudo demonstraram que apenas $58 \%$ dos inquiridos já implementaram o SCC, encontrando-se numa fase de implementação intermédia. Se atentarmos que a obrigatoriedade de implementação do SCC foi imposta em 2002, estes valores refletem uma taxa de adesão que se poderá considerar insuficiente (apesar da percentagem de implementação ter aumentado em relação a estudos anteriores).

Partilhamos da justificação avançada por Gomes et al. (2009), que refere que o atraso para a implementação do SCC pode estar relacionado com o facto da contabilidade de custos ser uma contabilidade interna, não estando a sua implementação sujeita a nenhum mecanismo de controlo nem a qualquer sanção por incumprimento, contrariamente ao que acontece com a contabilidade patrimonial e orçamental que são objeto de fiscalização por parte do Tribunal de Contas.

Além disso, considera-se que a especificidade e a complexidade do próprio SCC têm representado um entrave à implementação do mesmo. Carvalho et al. (2012) avançaram também que a utilização da informação produzida pelo SCC seria explicada de alguma forma pelo contexto organizacional interno (nomeadamente pela dificuldade na recolha e interpretação de dados disponibilizados pelo SCC) e pelo ambiente institucional (caracterizado pela perspetiva do isomorfismo). No mesmo sentido, Liguori (2012) conclui que a dinâmica intra-organizacional é fundamental para explicar e diferenciar os resultados de mudanças contábeis ocorridos.

Um dos fatores que mais contribuiu para a implementação do SCC foi a publicação de legislação, verificando-se, após a entrada em vigor do RGTAL e da LFL, um aumento dos municípios que aderiram à implementação do SCC. Esta situação permite-nos concluir que a implementação do SCC tem sido mais favorecida pela sua imposição como obrigação legal, do que propriamente pelo reconhecimento das mais-valias inerentes à implementação do sistema.

Contrariamente, os fatores que mais condicionaram a implementação do SCC foram a escassez dos recursos humanos, na percepção dos municípios não utilizadores, e a resistência por parte dos funcionários públicos ao controlo inerente ao SCC, na percepção dos municípios utilizadores. Em consonância, Scapens (1994) refere que as inovações contábeis são frequentemente sujeitas a resistência e as mudanças nos sistemas de contabilidade "travadas" mesmo que, obviamente contribuam para uma melhoria da tomada de decisão.

Confirmámos ainda a utilização pouco expressiva dos outputs produzidos pelo SCC, quer a nível interno na elaboração de documentos previsionais, quer a nível externo no reporte de informação, não se promovendo por esta via a accountability do município entre os diversos stakeholders. No mesmo sentido, Geiger e Ittner (1996) apontaram para o facto das entidades do setor público implementarem diferentes instrumentos de gestão para atender às exigências externas, mas fazerem pouco uso desses sistemas para fins internos. Outros 
estudos referem mesmo uma certa "apatia" para as inovações da gestão por parte do poder local (LAPSLEY; WRIGHT, 2004).

Por fim, confirma-se uma repercussão da informação produzida pelo SCC na melhoria da gestão do município e uma utilização da informação produzida pelo SCC, muito reduzida na maioria dos municípios (nomeadamente nos municípios de grande dimensão), não sendo esta situação explicada pela fase em que se encontrava a implementação do SCC. Deste modo, podemos concluir que, apesar dos utilizadores reconhecerem as mais-valias inerentes ao SCC, continuam a dar primazia, na tomada de decisão, às informações produzidas pelas demonstrações financeiras tradicionais. Em conformidade, Nogueira et al. (2013) avançam que os municípios continuam a dar primazia às informações das demonstrações financeiras tradicionais na tomada de decisão.

De forma a atenuar a utilização reduzida dos outputs produzidos pelo SCC, seria pertinente, para além da definição de uma estrutura concetual de custos (framework), um efetivo incentivo à disseminação dos conceitos inerentes ao SCC, para que os utilizadores percebam o alcance das informações produzidas e a apliquem na gestão municipal.

Quanto a limitações subjacentes a este estudo, assumimos que a técnica de recolha de dados utilizada, o inquérito por questionário, pode induzir a diferentes interpretações das questões aplicadas, em função das percepções de cada indivíduo.

Como recomendações para futuras investigações, consideramos de especial interesse a realização de uma pesquisa que considere a perspectiva diacrónica, possibilitando o estudo da evolução da problemática num período mais alargado, bem como a avaliação do impacto do contexto de austeridade em que o nosso país tem vivido.

\section{Referências}

BARRETTA, António; BUSCO, Cristiano. Technologies of government in public sector's networks: In search of cooperation through management control innovations. Management Accounting Research, v.22, p. 211-219, 2011.

BURNS, John; SCAPENS, Robert. Conceptualizing management accounting change: an institutional framework, Management Accounting Research, v.11, n. 1, p. 3-25, 2000.

CAIADO, António. Contabilidade Analítica ou de Gestão. Lisboa: Áreas Editora, 2012.

CAIADO, António; PINTO, Ana. Manual do plano oficial de contabilidade pública.

Lisboa: Áreas Editora, 2002.

CARDOSO, Ricardo; AQUINO, André; BITTI, Eugénio. Reflexões para um framework da informação de custos do setor público brasileiro. Revista de Administração Pública, v.45, n. 5, p. 1565-586, 2011.

CARVALHO, João; COSTA, Teresa; MACEDO, Natália. A contabilidade analítica ou de custos no setor público administrativo. Revista da Câmara dos Técnicos Oficiais de

Contas, v.96, p. 30-41, 2008. 
CARVALHO, João; FERNANDES, Maria; CAMÕES Pedro; JORGE, Susana. Anuário financeiro dos municípios portugueses 2011 e 2012. Lisboa: Ordem dos Técnicos Oficiais de Contas, 2013.

CARVALHO, João; GOMES, Patrícia e FERNANDES, Maria. The main determinants in the use of the Cost Accounting System in Portuguese local governments. Financial

Accountability and Management, v.28, n. 4, p. 60-88, 2012.

CARVALHO, João; JORGE, Susana e FERNANDES, Maria. Conformity and diversity of accounting and financial reporting practices in portuguese Local Government. Canadian Journal of Administrative Sciences, v.24, p. 2-14, 2007.

COSTA, Teresa. A utilidade da contabilidade de custos nas autarquias: o caso da fixação das tarifas e preços municipais. Dissertação de Mestrado - Universidade do Minho, Braga, 2005.

COSTA, Teresa C. S. O sistema da contabilidade de custos nas entidades locais em Portugal. in: Conferência Conocimiento, innovación y emprendedores; camino al futuro. Universidad de Rioja, 2007, España.

Decreto-Lei 46/1989, de 15 de fevereiro - Estabelece as matrizes de delimitação geográfica da Nomenclatura de Unidades Territoriais para Fins Estatísticos (NUTS).

Decreto-Lei n. ${ }^{o}$ 232/1997, de 3 de setembro - Aprova o Plano Oficial de Contabilidade Pública.

Decreto-Lei n. ${ }^{\circ}$ 54-A/1999, de 22 de fevereiro - Aprova o Plano Oficial de Contabilidade das Autarquias Locais.

EVANS, Patricia; BELLAMY, Sheila. Performance evaluation in the australian public sector - the role of management and cost accounting control systems. International Journal of Public Sector Management, v.6, p. 30-38, 1995.

FASAB. Managerial Cost Accounting Concepts and Standards for the Federal Government, SFFAS, n.4, 1995.

GEIGER, Dale; ITTNER, Christopher. The influence of funding source and legislative requirements on government cost accounting practices. Accounting, Organizations and Society, v.21, n.6, p. 549-567, 1996.

GOMES, Patrícia; CARVALHO, João; FERNANDES, Maria; Determinantes de adoção e desenvolvimento do sistema de contabilidade de custos nos municípios portugueses.

Contabilidade e Gestão, v.8, p. 11-40, 2009. 
GOMES, Patrícia; CARVALHO, João; FERNANDES, Maria. The main determinants of the use of the cost accounting system in Portuguese local government financial, Accountability \& Management, v.28, n.3, p. 306-334, 2012.

Van HELDEN, G.; AARDEMA, Harrie; BOGT, Henk; GROOT, Tom. Knowledge creation for practice in public sector management accounting by consultants and academics.

Preliminary findings and directions for future research. Management Accouting Research, v.21, p. 83-94, 2010.

HOOD, Christopher. Public management for all seasons?. Public Administration, v.69, n.1, p. 3-19, 1991

HOOD, Christopher. The 'new public management' in the 1980s: variations on a theme. Accounting, Organizations and Society, v.20, n.2/3, p. 93-109, 1995.

IGAE-Intervención General de la Administración del Estado. Contabilidad Analítica de las Administraciones Públicas. El Proyecto CANOA. Madrid: Lerko Print, 1994.

JACKSON, Audrey; LAPSLEY, Irvine. The diffusion of accounting practices in the new "managerial" public sector. International Journal of Public Sector Management, v.16, n.5, p. 359-372, 2003.

JOHNSON, Thomas; KAPLAN, Robert. Relevance lost: the rise and fall of management accounting. Boston: Harvard Business School Press, 1987.

KAPLAN, Robert; NORTON, David. The balanced scorecard - measures that drive performance. Harvard Business Review, Jan-Feb, v.71, n.9, 1992.

LAPSLEY, Irvine. New Public Management: The Cruellest Invention of the Human Spirit? Abacus, v.45, n.1, p. 1-21, 2009.

LAPSLEY, Irvine; WRIGHT, Elisa. The diffusion of management accounting innovations in the public sector: a research agenda. Management Accounting Research, v.15, p. 355-374, 2004.

Lei n. ${ }^{\circ}$ 8/1990, de 20 de fevereiro - Aprova a Lei de Bases da Contabilidade Pública.

Lei n. ${ }^{\circ}$ 53-E/2006, de 29 de dezembro - Aprova o Regime Geral de Taxas das Autarquias Locais.

Lei n. ${ }^{\circ}$ 2/2007 de 15 de janeiro - Aprova a Lei das Finanças Locais.

Lei n. ${ }^{\circ} 8 / 2012$, de 21 de fevereiro - Aprova a Lei dos Compromissos e Pagamentos em Atraso. 
Lei n. ${ }^{\circ}$ 75/2013, de 12 de setembro - Estabelece o Regime Jurídico das Autarquias Locais, Aprova o Estatuto das Entidades Intermunicipais, Estabelece o Regime Jurídico da Transferência de Competências do Estado para as Autarquias Locais e para as Entidades Intermunicipais e Aprova o Regime Jurídico do Associativismo Autárquico.

LIGUORI, Mariannnunziata. Radical change, accounting and public sector reforms: a comparison of italian and canadian municipalities. Financial Accountability \& Management, v.28(4), p. 0267-4424, 2012.

NOGUEIRA, Sónia; JORGE, Susana; Oliver, Mercedes. The usefulness of financial reporting for internal decision-making in Portuguese municipalities. Management Research: The Journal of the Iberoamerican Academy of Management, v.11, n.2, p. 178-212, 2013.

NYLAND, Kari, PETTERSEN, Inger. The control gap: the role of budgets, accounting information and (non) decisions in hospital settings. Financial Accountability \& Management, v.20, n.1, p. 77-102, 2004.

PEREIRA, Carlos; FRANCO, Victor. Contabilidade analítica. Lisboa: Rei dos Livros, 1994.

PETTERSEN, Inger. Implementing management accounting reforms in the public sector: the difficult journey from intentions to effects. The European Accounting Review, v.10, n.3, p. 561-581, 2001.

POLLITT, Christopher. Performance management in practice: a comparative study of executive agencies. Journal of Public Administration Researchand Theory, v.16, n. 1, p. 25-44, 2006.

POLLITT, Christopher. e BOUCKAERT, Geert. Public Management Reform: A Comparative Analysis. Oxford: Oxford University Press, 2004.

REGINATO, Elisabetta. Accountability perspectives in Italian municipality accounting systems: the gap between regulations and practices. Public Administration Quarterly, v.34, n. 4 , p. 552-590, 2010.

SANTOS, Sérgio. Modelo de imputação de custos e de recursos - o caso da Universidade do Minho. Revista Portuguesa de Educação, v.13, n.2, p. 267-292, 2000.

SATAPOCAL, Manual de Apoio Técnico à Aplicação do POCAL. Coimbra: Centro de Estudos e Formação Autárquica, 2006.

SCAPENS, Robert. Never mind the gap: towards an institutional perspective on management accounting practice. Management Accounting Research, v.5, p. 301-21, 1994. 